\title{
Determination of Cooling Rates in a Quadrupole Ion Trap
}

\author{
David M. Black, Anne H. Payne, and Gary L. Glish \\ Department of Chemistry, University of North Carolina at Chapel Hill, Chapel Hill, North Carolina, USA
}

\begin{abstract}
Collisional cooling rates of infrared excited ions are measured in a quadrupole ion trap (QIT) mass spectrometer at different combinations of temperature and pressure. Measurements are carried out by monitoring fragmentation efficiency of leucine enkephalin as a function of irradiation time by an infrared laser after a short excitation and incrementally increasing cooling periods. Cooling rates are observed to be directly related to bath gas pressure and inversely related to bath gas temperature. The cooling rate at typical ion trap operating pressure (1 mTorr) and temperature (room T) is faster than can be measured. At elevated temperature and the lowest pressure used for the studies, the rate of collisional cooling becomes negligible compared to the rate of radiative cooling. (J Am Soc Mass Spectrom 2006, 17, 932-938) (C) 2006 American Society for Mass Spectrometry
\end{abstract}

$\mathrm{F}$ or many years, collisional cooling has been used to damp the kinetic energies of trapped ions to improve the performance of the quadrupole ion trap mass spectrometer [1-3]. After formation in, or injection into, the quadrupole ion trap, ions undergo collisions with the bath gas, causing their trajectory to shrink to the center of the ion trap. This more compact cloud of ions increases sensitivity and resolution when using mass-selective instability [2] or resonance ejection [4] for mass analysis. A bath gas pressure of 1 mTorr is the commonly used operating pressure, and all commercial ion traps operate at ambient temperature.

Although collisional cooling of the ion's kinetic energy is helpful for ion trap performance, cooling of the ion's internal energy also occurs; this may or may not be desired. This internal cooling can reduce fragmentation efficiency and thus be a detriment when trying to dissociate ions using "slow heating" techniques [5] such as infrared multiphoton photodissociation (IRMPD). IRMPD has been successfully implemented in a quadrupole ion trap [6-15]. IRMPD works by increasing the internal energy of an ion by multiple photon absorption. When a bath gas pressure of 1 mTorr is used, the transfer of the ion's internal energy to the bath gas via collisions may occur at a faster rate than the rate of energy-transfer to the ions by photon absorption. The ratio of the rate of energy loss via collisional cooling to energy gain via photon absorption depends on the ion's absorption cross-section, the power of the laser, trapping volume temperature, and pressure. If the rate of

Published online May 12, 2006

Address reprint requests to Professor G. L. Glish, Kenan Laboratory, Department of Chemistry, University of North Carolina, Chapel Hill, NC 27599-3290, USA. E-mail: glish@unc.edu energy loss by collisions is greater than the rate of energy gained by photon absorption, the ions irradiated will not dissociate. It is due to this reason that IRMPD is not practical for peptide dissociation in a quadrupole ion trap at the typical operating pressure and roomtemperature [6, 15]. Most of the examples of IRMPD in the quadrupole ion trap have been accomplished by lowering the bath gas pressure. At decreased pressures, however, the performance of the QIT is adversely affected. Sensitivity is decreased by approximately an order of magnitude [6], and resolution is also decreased.

An alternate approach to overcome the problem of collisional cooling is thermally assisted infrared multiphoton photodissociation (TA-IRMPD) [6]. In the TAIRMPD experiment, the bath gas and quadrupole ion trap electrodes are heated, which reduces the rate of collisional cooling. TA-IRMPD has been used to achieve high IRMPD fragmentation efficiencies at typical bath gas pressures ( $1 \mathrm{mTorr})$. While the ion being dissociated will initially have greater internal energy when the bath gas is heated versus ambient bath gas temperature, the more important effect in the success of TA-IRMPD can be attributed to less energy being transferred per collision between an IR-excited ion and a bath gas atom. The amount of energy transferred per collision is directly related to the difference in total energy between the ion and bath gas molecule. A theoretical treatment of collisional cooling [16] reports the use of an average down step size (i.e., energy lost per collision) as $\alpha=$ $0.625 k \mathrm{~T}+0.001 k\left(\mathrm{~T}_{\text {int }}-\mathrm{T}\right)$, where $\mathrm{T}$ and $\mathrm{T}_{\text {int }}$ are the temperature of the bath gas and temperature that describes the distribution of internal energies of the ions, respectively.

A few previous experimental and theoretical studies of collisional cooling in the QIT mass spectrometer have 
been performed. In one study, theoretical cooling calculations for ions larger than $1 \mathrm{kDa}$ were performed [17]. Two models, the diffuse scattering model and the random walk model, were used to bracket the range of ion cooling rates. The calculations were done assuming helium as the bath gas at $1 \mathrm{mTorr}$ and room temperature. Another study reported the experimental collisional cooling of ion kinetic energy [18]. Collisional cooling was studied qualitatively at different pressures by allowing increasing amounts of cool time after ion injection or resonance excitation followed by mass analysis. Ion current was plotted as a function of cool time and was seen to increase with increasing cool time. Other parameters such as pressure and $\mathrm{q}_{\mathrm{z}}$ were also varied to observe their affect on cooling rate. In contrast to the limited studies of ion cooling in a QIT mass spectrometer, there are many studies of rates of radiative and collisional cooling in ion cyclotron resonance mass spectrometers. Many methods for cooling rate determination have been reported [19]. To determine internal energy cooling rates in a QIT mass spctrometer, we used a procedure similar to the two-pulse photodissociation experiments reported previously [20-22].

Determination of rates of collisional cooling as well as the relationship between pressure and cooling rate will be beneficial in understanding the kinetics of energy gain and loss through photon absorption and collisions of an excited ion with a bath gas. By varying temperature, it is also possible to observe the relationship between collisional cooling rate and trapping volume temperature. In this study, we demonstrate a method to measure collisional cooling rates of infrared excited ions in a QIT mass spectrometer. Because the ions are cooled kinetically before excitation, and infrared photons are being used to excite the ions, it can be assumed that the collisional cooling rate measured represents only the loss of the ions' internal energy.

\section{Experimental}

Experiments were carried out using a highly modified Finnigan ITMS (San Jose, CA) with a custom-built nanoelectrospray source. ICMS software was used for the control of the instrument [23]. Leucine enkephalin (YGGFL) was purchased from Sigma Chemical Company (St. Louis, MO) and was used to prepare $100 \mu \mathrm{M}$ solutions in 75:20:5 $\mathrm{CH}_{3} \mathrm{OH}: \mathrm{H}_{2} \mathrm{O}: \mathrm{CH}_{3} \mathrm{COOH}$. Trapping volume pressures of helium used for experiments range from $2.8 \times 10^{-4}$ torr to $1.0 \times 10^{-3}$ torr. Pressure was measured using an ionization gauge and correction factors were applied for helium. Temperatures range from ambient temperature, $\sim 25^{\circ} \mathrm{C}$, to $165^{\circ} \mathrm{C}$. A $1000 \mathrm{~W}$ stab-in bake out heater located under the quadrupole ion trap electrodes is used to heat the electrodes and bath gas. The temperature is measured with a platinum resistance thermometer located touching one of the endcap electrodes. A Synrad 50 Watt IR $(10.6 \mu \mathrm{m}$, Mukilteo, WA) continuous wave laser was used for excitation and dissociation of protonated leucine en-

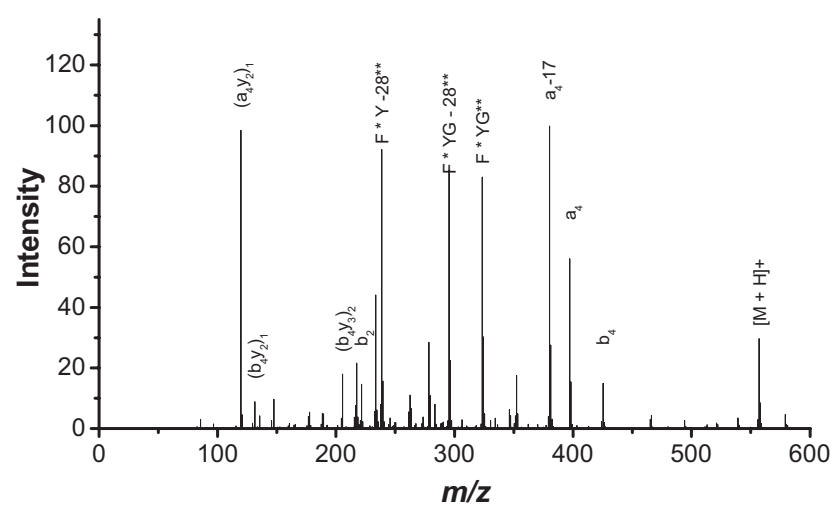

Figure 1. IRMPD of $\left[\right.$ YGGFL $+\mathrm{H}^{+}$with $30 \mathrm{~ms}$ irradiation time.

kephalin. The laser beam enters the vacuum housing through a ZnSe window on the side of the vacuum housing. The ring electrode of the quadrupole ion trap is modified with a $3.2 \mathrm{~mm}$. hole through the center, perpendicular to the axial direction. The laser beam is oriented so that it passes through this hole and into the trapping volume in the radial direction. A TTL pulse from the ITMS electronics that is controlled by the ICMS software triggers the laser. Laser output is set to $100 \%$ $(50 \mathrm{~W})$. Each data point represents an average of ten scans.

Leucine enkephalin was used for all cooling studies. The ions of $\mathrm{m} / z 556[\mathrm{M}+\mathrm{H}]^{+}$are isolated in the quadrupole ion trap by ramping the radio frequency (rf) amplitude applied to the ring electrode to eject all ions with a $\mathrm{m} / \mathrm{z}$ of less than 556 . Additionally, a supplementary AC voltage is applied to the endcap electrodes with a frequency initially corresponding to the secular frequency of $m / z 557$. Ions with $m / z$ values greater than 556 are ejected as the rf amplitude is increased and they come into resonance with the supplementary AC voltage.

Cooling curve plots are made by plotting fragmentation efficiency as a function of laser time. Separate plots are made for each combination of temperature and pressure. For each plot, a three-step procedure was used. First, the laser is used to excite (but not dissociate) the ions stored in the quadrupole ion trap (excitation IR). For all combinations of temperature and pressure, a $5 \mathrm{~ms}$ pulse of the laser is used to excite the ions. During the second step (cool time), the ions are allowed to cool. The time that the ions are allowed to cool is incrementally increased from $0 \mathrm{~ms}$ to the amount of time needed for the ions to return to the initial state of equilibrium with the helium bath gas. During the third step, the ions are dissociated with a second irradiation period by the laser (dissociation IR). The dissociation IR time is incrementally increased from $0 \mathrm{~ms}$ to the amount of time needed to observe a leveling off of the fragmentation efficiency. A control experiment is also run to determine fragmentation efficiencies as a function of laser time without prior excitation. The control experiment in- 
cludes only step three of the above procedure (i.e., neither excitation IR nor cool time is used in the control experiment).

All product ions in the IRMPD MS/MS spectrum of leucine enkephalin shown in Figure 1 were used for fragmentation efficiency calculations. It has previously been shown that a major pathway in the dissociation of leucine enkephalin is a complex rearrangement where the C-terminal residue from an $\mathrm{a}_{4}{ }^{+}$ion is transferred to the $\mathrm{N}$-terminus and the new $\mathrm{C}$-terminal residue is subsequently lost $\left(\mathrm{YGGF}^{+} \rightarrow \mathrm{FYGG}^{+} \rightarrow \mathrm{FYG}^{+}\right)$[24]. Ions that are the result of this rearrangement and subsequent dissociation are indicated by one-letter abbreviations corresponding to the residues making up the product ion. The linkage between the transferred residue to the $\mathrm{N}$-terminus is marked with an asterisk $\left(^{*}\right)$. All other product ions are labeled ions are labeled using conventional nomenclature $[25,26]$.

Because cooling curves are plots of fragmentation efficiency as a function of laser irradiation time, sources of error are related to parameters that can affect the fragmentation efficiency from scan to scan. Differences from scan to scan in the number of ions stored in the quadrupole ion trap should not affect the fragmentation efficiency if we assume the photon to ion ratio to be very large. The main contributor to any source of error would most likely be due to fluctuations in laser power from one laser pulse to the next. To minimize the effects of laser power fluctuation, each data point shown in the cooling curves represents an average of 10 scans and all data for a combination of temperature and pressure were taken contiguously. The maximum error in cooling rates, determined using $95 \%$ confidence intervals is less than $30 \%$ for all combinations of temperature and pressure.

When using IRMPD to excite the ions held in the quadrupole ion trap, it is not necessary to tune any parameters, such as $\mathrm{q}_{\mathrm{z}}$, voltage, or frequency, before irradiation. Only the laser beam alignment through the quadrupole ion trap must be consistent to achieve similar fragmentation efficiencies from day to day. For these studies, the laser was not aligned so that the laser beam went directly through the center of the quadrupole ion trap but instead was reflected inside the quadrupole ion trap. By not aligning the laser directly through the center of the quadrupole ion trap, higher fragmentation efficiency could be achieved in shorter times. This results in steeper slopes of the fragmentation efficiency curves (vide infra) and less error in the measurement.

\section{Theory}

Figure 2 shows schematically the expected results from the experiment to measure cooling rates. After the initial IR activation, as the cool time is increased the corresponding fragmentation efficiency curve shifts to the right towards the control curve, which is to the far right. The fragmentation efficiency is defined in eq 1.

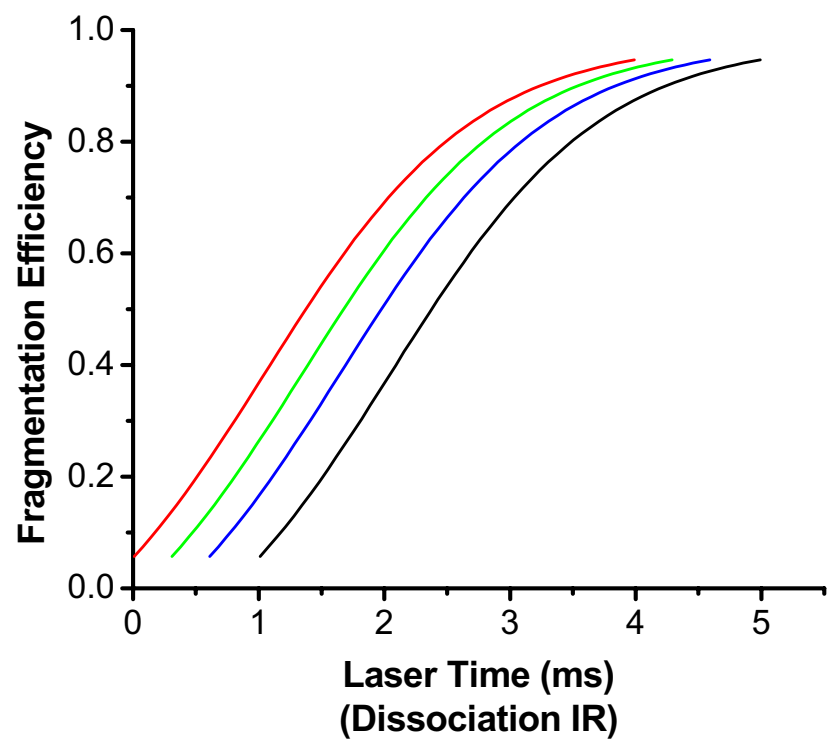

Figure 2. Hypothetical cooling curve plot. With increasing cool time curves shift to longer dissociation IR times indicating increased cooling. The red line represents data taken with an excitation IR pulse, no cool time between the first pulse and a second incrementally increasing IR pulse. The green and blue lines represent data taken with an excitation IR pulse, followed by a cool time and incrementally increasing second IR pulse. The cool time is longer for the blue line than that for the green line. The black line represents data taken with no excitation IR pulse and no cool time but only the incrementally increasing IR pulse time (control curve).

$$
\text { Fragmentation Efficiency }=\frac{\sum \text { products }}{\sum \text { parent }+ \text { products }}
$$

The shift of the curve to the right represents the loss of a fraction of the initial internal energy gained by the ion from the excitation IR. When the cool time is long enough so that all internal energy gained by the ions from the excitation IR is lost through collisions and radiative relaxation, the dissociation IR curve for that cool time will overlap with the control curve. The loss of energy obtained from the excitation IR, or cooling, can be represented by plotting fragmentation efficiency with respect to the dissociation IR time (second irradiation) for each cool time increment. Cooling rates can be determined by monitoring a change in fragmentation efficiency as the cool time is increased. It is not necessary for the ions to cool to "thermal equilibrium" with the bath gas for determination of cooling rates.

To determine a cooling rate at a given temperature and pressure, the average fragmentation efficiency corresponding to a range of dissociation IR times was calculated for each curve. The range of dissociation IR times used to calculate the average, indicated by the box in Figure 3, is chosen so that each point contributing to the average is located at a point in the curves where fragmentation efficiency is increasing and has not leveled off. The purpose for this is that by averaging over a range of dissociation IR times, the effect of any minor 


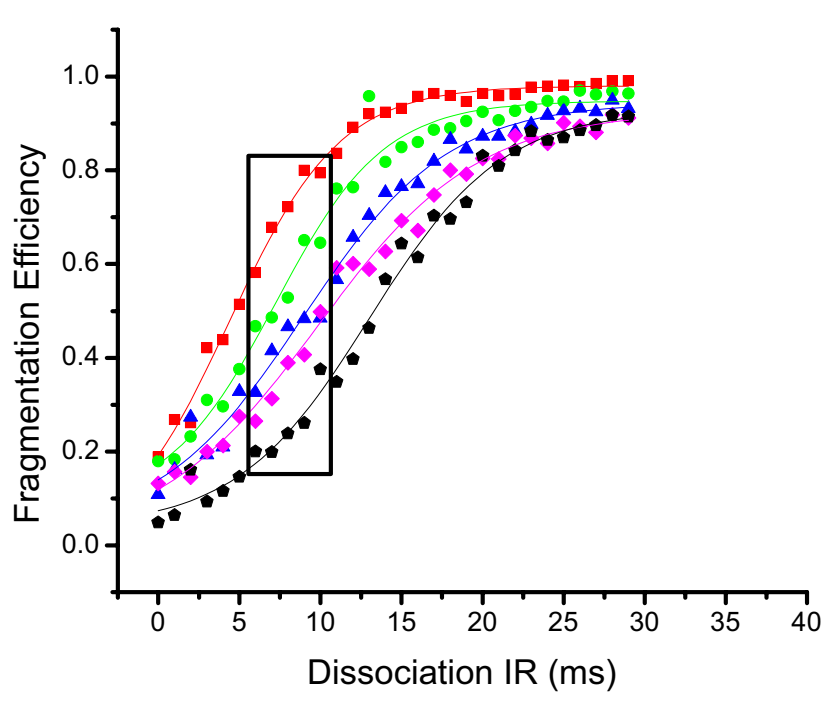

Figure 3. Cooling curves recorded at $165^{\circ} \mathrm{C}$ and $2.45 \times 10^{-4}$ torr. Box indicates dissociation IR range used to calculate average fragmentation efficiency for each curve. Red square, green circle, blue triangle, purple diamond, and black pentagon shaped points represent data taken with $5 \mathrm{~ms}$ excitation IR $0 \mathrm{~ms}$ cool time, $5 \mathrm{~ms}$ excitation IR $6 \mathrm{~ms}$ cool time, $5 \mathrm{~ms}$ excitation IR $9 \mathrm{~ms}$ cool time, 5 $\mathrm{ms}$ excitation IR $12 \mathrm{~ms}$ cool time, and $0 \mathrm{~ms}$ excitation IR $0 \mathrm{~ms}$ cool time, respectively.

differences in the shapes of curves on the cooling rate should be minimized. Average fragmentation efficiency is then plotted as a function of cool time. The decrease in average fragmentation efficiency with increasing cool time is directly related to a decrease in the population of vibrationally excited leucine enkephalin. A linear fit to the data is then used to determine the slope and error for the slope of the fitted line. The slope corresponds to the pseudo first-order cooling rate constant.

To determine the cooling rate constants several assumptions are made with respect to the processes occurring in the quadrupole ion trap. Several mechanisms of energy-transfer can occur and are shown by the Lindemann-Hinshelwood mechanisms below where LE and He represent leucine enkephalin and helium respectively.

$$
\begin{aligned}
& \mathrm{LE}^{+}+\mathrm{He} \stackrel{k_{\text {excite }}}{\rightarrow} \mathrm{LE}^{+} *+\mathrm{He} \\
& L E^{+}+H e \stackrel{k_{\text {ccool }}}{\leftarrow} L E^{+*}+H e \\
& \mathrm{LE}^{+*} \stackrel{k_{\text {dissociation }}}{\rightarrow} \text { products } \\
& L E^{+*} \stackrel{k_{\text {radiative }}}{\rightarrow} L E^{+}+h v
\end{aligned}
$$

These equations can summed up as the overall rate equation:

$$
\begin{aligned}
& \frac{d\left[L E^{+*}\right]}{d t}=k_{\text {excite }}[\mathrm{He}]\left[L E^{+}\right]-k_{\text {ccool }}[\mathrm{He}]\left[L E^{+*}\right] \\
& -k_{\text {dissociation }}\left[L E^{+*}\right]-k_{\text {radiative }}\left[L E^{+*}\right]
\end{aligned}
$$

$\mathrm{k}_{\text {ccool }}, \mathrm{k}_{\text {excite, }} \mathrm{k}_{\text {dissociation }}$ and $\mathrm{k}_{\text {radiative }}$ represent the second-order cooling rate constant, second-order excitation/collisional heating rate constant, the first-order unimolecular dissociation rate constant and the firstorder radiative cooling rate constant, respectively. Eqs 2 and 3 indicate processes of collisional excitation and cooling that occur in the quadrupole ion trap due to collisions of the ions with the helium bath gas. No resonance excitation was used for these experiments so contributions from collisional excitation are insignificant and can be removed from the overall rate equation. $\mathrm{Eq} 4$ represents the process of unimolecular dissociation of activated leucine enkephalin ions. Under the conditions used for these experiments the unimolecular dissociation of leucine enkephalin occurs at a rate that is much too slow to be observed under all combinations of temperature and pressure used for these experiments. Additionally, because the first irradiation by the laser ( 5 $\mathrm{ms}$ ) is selected so that the ions are only excited but not dissociated, it can be assumed that no leucine enkephalin is dissociated by this first irradiation. As a result, the contribution to the overall rate equation from the loss of excited leucine enkephalin due to dissociation can also be assumed to be insignificant and removed from the overall equation.

By removing terms due to excitation and dissociation, the overall equation simplifies to:

$$
\frac{d\left[L E^{+*}\right]}{d t}=-k_{\text {ccooo }}[\mathrm{He}]\left[L E^{+*}\right]-k_{\text {radiative }}\left[L E^{+*}\right]
$$

The only processes that contribute to the decrease in excited leucine enkephalin for the purposes of our experiment are those due to cooling via collisions with the bath gas as well as cooling due to emission of radiation. The equation can be simplified even more since the number of helium atoms in the quadrupole ion trap is much greater than the number of excited-state leucine enkephalin ions. As a result, the second-order overall rate equation can be reduced to a pseudo first-order equation so that it then becomes the following (eq 8) where $\mathrm{k}_{\text {ccool }}^{\prime}$ is defined in eq 9:

$$
\begin{aligned}
& \frac{d\left[L E^{+*}\right]}{d t}=-k_{\text {ccool }}^{\prime}\left[L E^{+*}\right]-k_{\text {radiative }}\left[L E^{+*}\right] \\
& k_{\text {ccool }}^{\prime}=k_{\text {ccool }}[\mathrm{He}]
\end{aligned}
$$

For most of the combinations of temperature and pressure used for this study, both radiative and collisional cooling contribute to the overall cooling rate. However, at higher temperatures and lower pressures, collisional cooling is slow enough so that cooling due to radiative emission becomes predominant. Collisional cooling rates can be determined by subtracting the rate of radiative cooling, determined from the experiments at high-temperature and low-pressure, from the overall cooling rate measured for each combination of temper- 

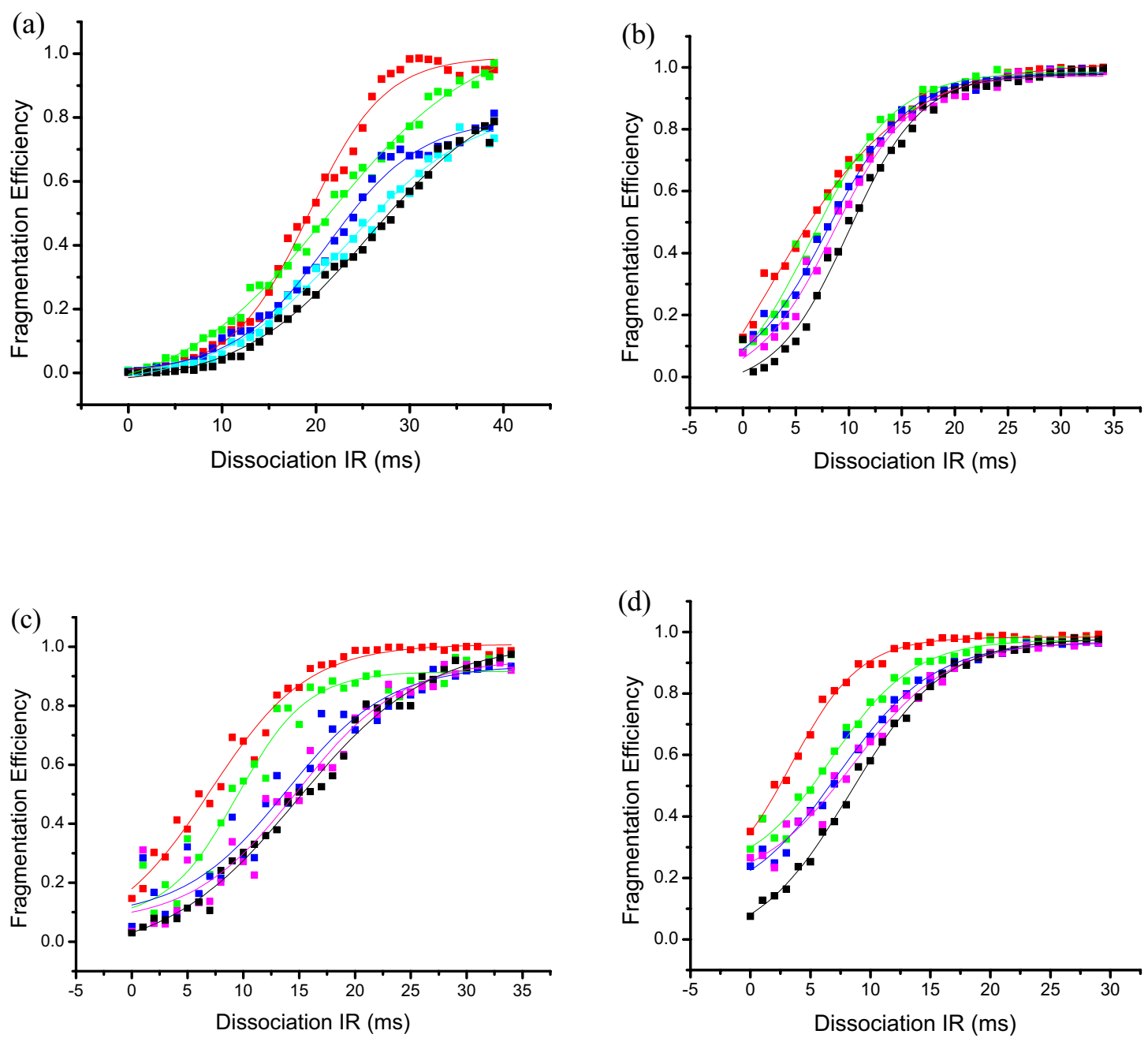

Figure 4. Cooling curves recorded at $2.80 \times 10^{-4}$ torr and (a) $25^{\circ} \mathrm{C}$, (b) $100{ }^{\circ} \mathrm{C}$, (c) $135^{\circ} \mathrm{C}$, and (d) $165^{\circ} \mathrm{C}$.

ature and pressure. This assumes radiative emission is independent of pressure and temperature over the ranges that were studied.

\section{Results and Discussion}

For all combinations of temperature and pressure studied, the cooling of the IR excited ions to "thermal equilibrium" with the bath gas occurred within $20 \mathrm{~ms}$. Because collisional cooling occurs relatively rapidly in a quadrupole ion trap at standard operating pressures, only combinations of temperature and pressure for which cooling occurred within times between 5 and 20 $\mathrm{ms}$ were candidates for cooling measurements. When cooling occurs over shorter intervals than these, cooling curve measurements become complicated by decreased fragmentation efficiencies even with extensive irradiation times. Cooling intervals longer than $20 \mathrm{~ms}$ were not observed at the temperatures and pressures studied. Unfortunately, the cooling rate could not be slowed to lower rates because of very weak ion signal with pressures lower than $2.80 \times 10^{-4}$ torr and a maximum trapping volume heating temperature of $\sim 165^{\circ} \mathrm{C}$.

Cooling rate measurements were made at pressures of $2.80 \times 10^{-4}, 3.15 \times 10^{-4}, 3.50 \times 10^{-4}, 3.85 \times 10^{-4}$, and $4.20 \times 10^{-4}$ torr. Temperatures used for cooling measurements include $25^{\circ} \mathrm{C}, 100^{\circ} \mathrm{C}, 135^{\circ} \mathrm{C}$, and $165^{\circ} \mathrm{C}$. At bath gas pressures of $2.80 \times 10^{-4}$ torr, collisional cooling rates are low enough to allow for efficient dissociation and cooling rate determination at all temperatures investigated. Figure 4 shows cooling curve data taken at $2.80 \times 10^{-4}$ torr at four different temperatures. All data for a given temperature was taken on the same day. However, data taken at different temperatures were done so on different days and, therefore, were subject to differences in laser alignment. Due to these differences in laser alignment, the shape of each set of curves and irradiation time needed for the fragmentation efficiency to level off at $100 \%$ for curves at different temperatures cannot be compared.

All curves have been fitted with a Boltzmann sigmoidal growth function for the purposes of visual aid only. 


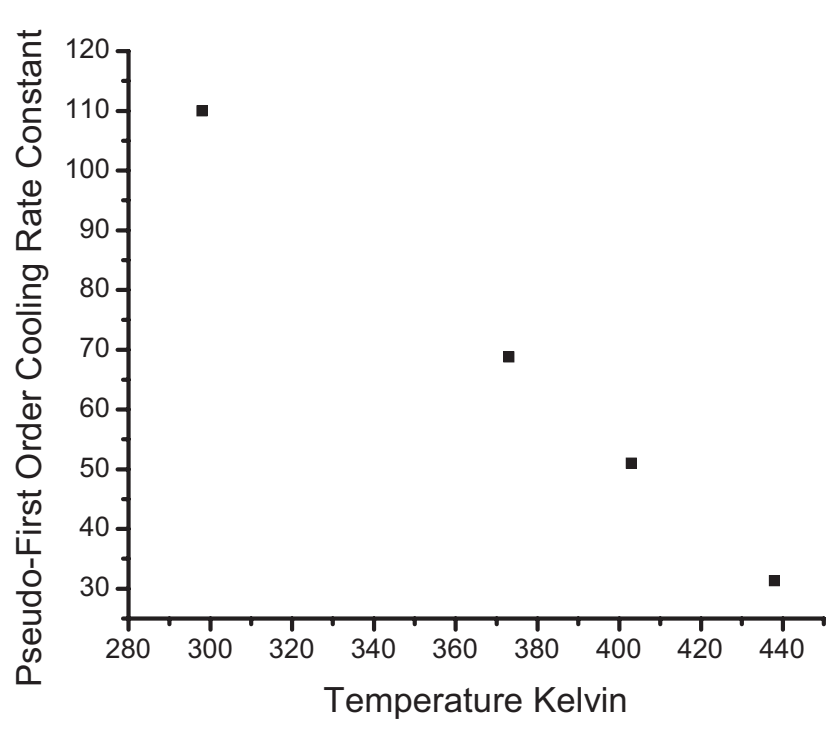

Figure 5. Plot of pseudo first-order cooling rate constant as a function of temperature at $2.80 \times 10^{-4}$ torr.

As can be seen in the plots, as the cool time is increased, the fragmentation efficiency curve shifts to the right. This indicates that a longer dissociation IR time is needed to achieve the same degree of dissociation at longer cool times between the excitation IR and dissociation IR than was needed with shorter cool times. Figure $4 \mathrm{a}, \mathrm{b}, \mathrm{c}$, and $\mathrm{d}$ show data taken at $2.80 \times 10^{-4}$ torr and $25^{\circ} \mathrm{C}, 100^{\circ} \mathrm{C}, 135^{\circ} \mathrm{C}$, and $165^{\circ} \mathrm{C}$, respectively. The cooling time increments for these temperatures are 2, 3, 5 , and $5 \mathrm{~ms}$ for $25^{\circ} \mathrm{C}, 100^{\circ} \mathrm{C}, 135^{\circ} \mathrm{C}$, and $165^{\circ} \mathrm{C}$, respectively. In Figure 4a, which represents data taken at $25^{\circ} \mathrm{C}$, the energy from the $5 \mathrm{~ms}$ excitation burst is lost through cooling processes within $\sim 6 \mathrm{~ms}$. At $100{ }^{\circ} \mathrm{C}$, $135^{\circ} \mathrm{C}$, and $165^{\circ} \mathrm{C}$, cooling is completed in $\sim 12,15$, and $20 \mathrm{~ms}$, respectively. A plot of the pseudo first-order rate constant versus temperature at $2.80 \times 10^{-4}$ torr is shown in Figure 5. As the temperature is increased, the pseudo first-order cooling rate decreases linearly over the range of temperatures studied.

At the highest temperature used for the study, $165^{\circ} \mathrm{C}$, the cooling rate was measured at six pressures between $2.45 \times 10^{-4}$ torr to $4.20 \times 10^{-4}$ torr. Over this small pressure range the second-order rate constants do not change significantly (Table 1). However, there is a much greater change in the pseudo first-order rage constants. A plot of the pseudo first-order rate constant as a function of pressure is shown in Figures 6. As the pressure is decreased, a decrease in the rate of cooling is observed down to a pressure of $3.15 \times 10^{-4}$ torr. Below a pressure of $3.15 \times 10^{-4}$ torr the pseudo first-order cooling rate levels off to a rate of $\sim 30 \mathrm{~s}^{-1}$. This is indicative of a decrease in the rate of collisional cooling

Table 1. Second order cooling rate constants $\left(\mathrm{cm}^{3} \mathrm{~mol}^{-1} \mathrm{sec}^{-1}\right)$ at $165^{\circ} \mathrm{C}$

\begin{tabular}{lllllll}
\hline Pressure $\left(\times 10^{-4}\right)$ & 2.45 & 2.80 & 3.15 & 3.50 & 3.85 & 4.20 \\
Rate Constant $\left(\times 10^{-12}\right)$ & 5.73 & 5.07 & 4.80 & 6.00 & 7.06 & 10.6 \\
\hline
\end{tabular}

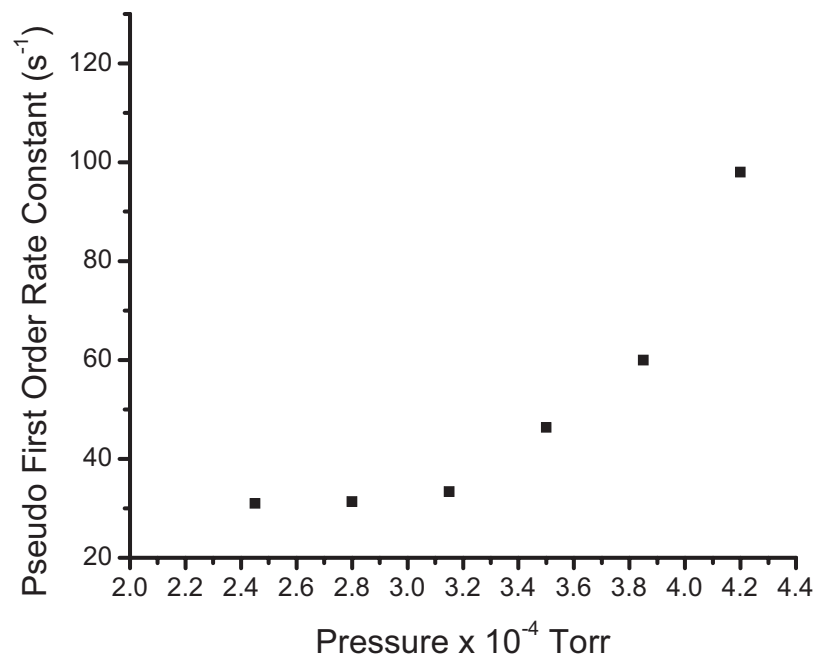

Figure 6. Plot of pseudo first-order cooling rate constant as a function of pressure at $165^{\circ} \mathrm{C}$.

to a point where it is no longer a significant contributor to the overall cooling of the ions. Below this point, collisional cooling is a minor component to the overall cooling rate and radiative cooling becomes predominant (see eq 8).

\section{Conclusions}

Collisional cooling to damp the kinetic energy of ions in a quadrupole ion trap mass spectrometer is important in improving the sensitivity and resolution. However, internal energy cooling also occurs, which can be detrimental when trying to dissociate ions by slow heating methods such as IRMPD. The overall cooling rate is a combination of collisional and radiative cooling. Collisional and radiative cooling rates in a quadrupole ion trap can be determined using the method described in this work. At $2.80 \times 10^{-4}$ torr, pseudo first-order cooling rates decrease from $\sim 110 \mathrm{~s}^{-1}$ at $25^{\circ} \mathrm{C}$ to $\sim 30 \mathrm{~s}^{-1}$ at $165^{\circ} \mathrm{C}$. The pseudo first-order cooling rates are shown to decrease as pressure is lowered and reach a minimum of $\sim 30 \mathrm{~s}^{-1}$ at pressures below $3.15 \times 10^{-4}$ torr at $165{ }^{\circ} \mathrm{C}$. This is the radiative cooling rate at this temperature.

\section{Acknowledgments}

This work was supported by NIH grant GM49852.

\section{References}

1. March, R. E.; Londry, F. A. In Practical Aspects of Ion Trap Mass Spectrometry; March, R. E.; Todd, J. F. J., Eds.; CRC Press: Boca Raton, 1995; pp 25-48.

2. Stafford, G. C. J.; Kelley, P. E.; Syka, J. E. P.; Reynolds, W. E.; Todd, J. F. J. Recent Improvements in and Analytical Applications of Advanced Ion Trap Technology. Int. J. Mass Spectrom. Ion Processes 1984, 60, 85-98.

3. Goeringer, D. E.; McLuckey, S. A. Evolution of Ion Internal Energy During Collisional Excitation in the Paul Ion Trap: A Stochastic Approach. J. Chem. Phys. 1996, 104, 2214-2221.

4. Fulford, J. E.; Hoa, D. N.; Hughes, R. J.; March, R. E.; Bonner, R. F.; Wong, G. J. Radio-Frequency Mass Selective Excitation and Resonant 
Ejection of Ions in a Three-Dimensional Quadrupole Ion Trap. J. Vac. Sci. Technol. 1980, 17, 829-835.

5. McLuckey, S. A. Principles of Collisional Activation in Analytical Mass Spectrometry. J. Am. Soc. Mass Spectrom. 1992, 3, 599-614.

6. Payne, A. H.; Glish, G. L. Thermally Assisted Infrared Multiphoton Photodissociation in a Quadrupole Ion Trap. Anal. Chem. 2001, 73, 3542-3548.

7. Stephenson, J. L.; Booth, M. M.; Shalosky, J. A.; Eyler, J. R.; Yost, R. A. Infrared Multiple Photon Dissociation in the Quadrupole Ion Trap Via a Multipass Optical Arrangement. J. Am. Soc. Mass Spectrom. 1994, 5, 886-893.

8. Colorado, A.; Shen, J. X.; Vartanian, V. H.; Brodbelt, J. S. Use of Infrared Multiphoton Photodissociation with SWIFT for Electrospray Ionization and Laser Desorption Applications in a Quadrupole Ion Trap Mass Spectrometer. Anal. Chem. 1996, 68, 4033-4043.

9. Goolsby, B. J.; Brodbelt, J. S. Characterization of b-Lactams by Photodissociation and Collision-Activated Dissociation in a Quadrupole Ion Trap. J. Mass Spectrom. 1998, 33, 705-712.

10. Goolsby, B. J.; Brodbelt, J. S. Analysis of Protonated and Alkali Metal Cationized Aminoglycoside Antibiotics by Collision-Activated Dissociation and Infrared Multi-Photon Dissociation in the Quadrupole Ion Trap. J. Mass Spectrom. 2000, 35, 1011-1024.

11. Boue, S. M.; Stephenson, J. L.; Yost, R. A. Pulsed Helium Introduction into a Quadrupole Ion Trap for Reduced Collisional Quenching During Infrared Multiphoton Dissociation of Electrosprayed Ions. Rapid Commun. Mass Spectrom. 2000, 14, 1391-1397.

12. Crowe, M. C.; Brodbelt, J. S.; Goolsby, B. J.; Hergenrother, P. Characterization of Erythromycin Analogs by Collisional Activated Dissociation and Infrared Multiphoton Dissociation in a Quadrupole Ion Trap. J. Am. Soc. Mass Spectrom. 2002, 13, 630-649.

13. Hashimoto, Y.; Hasegawa, H.; Yoshinari, K.; Waki, I. Collision-Activated Infrared Multiphoton Dissociation in a Quadrupole Ion Trap Mass Spectrometer. Anal. Chem. 2003, 75, 420-425.
14. Keller, K. M.; Brodbelt, J. Collisionally Activated Dissociation and Infrared Multiphoton Dissociation of Oligonucleotides in a Quadrupole Ion Trap. Anal. Biochem. 2004, 326, 200-210.

15. Crowe, M. C.; Brodbelt, J. Infrared Multiphoton Dissociation (IRMPD) and Collisionally Activated Dissociation of Peptides in a Quadrupole Ion Trap with Selective IRMPD of Phosphopeptides. J. Am. Soc. Mass Spectrom. 2004, 15, 1581-1592.

16. Asano, K. G.; Goeringer, D. E.; McLuckey, S. A. Thermal Dissociation in the Quadrupole Ion Trap: Ions Derived from Leucine Enkephalin. Int. J. Mass Spectrom. 1999, 185/186/187, 207-219.

17. Goeringer, D. E.; McLuckey, S. A. Relaxation of Internally Excited High-Mass Ions Simulated under Typical Quadrupole Ion Trap Storage Conditions. Int. J. Mass Spectrom. 1998, 177, 163-174.

18. Wu, H. F.; Brodbelt, J. S. Effects of Collisional Cooling on Ion Detection in a Quadrupole Ion Trap Mass Spectrometer. Int. J. Mass Spectrom. Ion Processes 1992, 115, 67-81.

19. Dunbar, R. C. Infrared Radiative Cooling of Gas-Phase Ions. Mass Spectrom. Rev. 1992, 11, 309-339.

20. Freiser, B. S.; Beauchamp, J. L. Laser Photodissociation of Benzene Radical Cations. Evidence for a Two Photon Process Involving a Long Lived Intermediate. Chem. Phys. 1975, 35, 35-40.

21. Faulk, J. F.; Dunbar, R. C. Ion Cyclotron Resonance Ion Trap Measurements of Energy Relaxation in Gas-Phase Ions: Three Techniques Compared for Thiophenol Ion. J. Phys. Chem. 1989, 93, 7785-7789.

22. Asamoto, B.; Dunbar, R. C. Observation of the Infrared Radiative Relaxation of Iodobenzene Ions Using Two-Light-Pulse Photodissociation. J. Phys. Chem. 1987, 91, 2804-2807.

23. Yates, N.; Yost, R. ICMS Ion Trap Software 2.20

24. Vachet, R. W.; M., B. B.; Erickson, B. W.; Glish, G. L. Novel Peptide Dissociation: Gas-Phase Intramolecular Rearrangement of Internal Amino Acid Residues. J. Am. Chem. Soc. 1997, 119, 5481-5488.

25. Roepstorff, P. Proposal for a Common Nomenclature for Sequence Ions in Mass Spectra of Peptides. Biomed. Mass Spectrom. 1984, 11, 601.

26. Biemann, K. Contributions of Mass Spectrometry to Peptide and Protein Structure. Biomed. Environ. Mass Spectrom. 1988, 16, 99-111. 